\title{
Carnets
}

Revue électronique d'études françaises de l'APEF

Première Série - 1 Numéro Spécial | 2009

Cultures littéraires : nouvelles performances \& développement

\section{Les ressorts fictionnels d'une ville. Le cas de Naples}

\section{Nathalie Roelens}

\section{CpenEdition}

Journals

Édition électronique

URL : http://journals.openedition.org/carnets/4356

DOI : $10.4000 /$ carnets.4356

ISSN : 1646-7698

Éditeur

APEF

Édition imprimée

Date de publication : 1 juin 2009

Pagination : 433-450

\section{Référence électronique}

Nathalie Roelens, "Les ressorts fictionnels d'une ville. Le cas de Naples », Carnets [En ligne], Première Série - 1 Numéro Spécial | 2009, mis en ligne le 16 juin 2018, consulté le 01 mai 2019. URL : http:// journals.openedition.org/carnets/4356 ; DOI : 10.4000/carnets.4356

\section{(c) (i) ()ㅜㄹ}

Carnets est mis à disposition selon les termes de la licence Creative Commons - Atribution - Pas d'utilisation commerciale 4.0 International. 


\title{
LES RESSORTS FICTIONNELS D'UNE VILLE \\ Le cas de Naples
}

Nathalie Roelens

Université de Nimègue

\begin{abstract}
Résumé
Souvent les écrivains contribuent à façonner l'imaginaire d'une ville. La vivacité culturelle de Naples se mesure en effet à l'intérêt qu'elle a soulevé et aux « mises en discours » qu'elle a suscitées. Cette destination méridionale a toujours attiré les écrivains nordiques comme un îlot de liberté propice à la licence poétique. Montesquieu ou Dumas d'abord contrariés et ensuite fascinés par l'imposture du miracle de San Gennaro, le Marquis de Sade en quête d'écarts inédits, Stendhal avide d'opéra lyrique, et plus récemment Jean-Noël Schifano rédigeant un Dictionnaire amoureux, ces auteurs ont chacun à leur façon réinventé la ville. C'est entre un scepticisme initial et une adulation quasi idolâtre que se déploie l'imaginaire de Naples, nourrie de textes, d'Histoire, de produits culturels. Si une ville peut être réhabilitée par des textes, nous pouvons arguer que la littérature possède encore une certaine valeur et une certaine légitimité.
\end{abstract}

\begin{abstract}
Often writers have contributed to shape the imaginary of a city. The cultural vivacity of Naples can indeed be measured by the interest that it has aroused and by the "discourses " it has generated. This southern destination has always attracted northern writers as an islet of freedom inclined to poetic licence. Montesquieu or Dumas, first perplex and then fascinated by of the imposture of San Gennaro's miracle, the Marquis de Sade using his own travel guide as a pretext for unseen depravity, Stendhal eager for lyric opera, and more recently Jean-Noël Schifano writing a Dictionnaire amoureux de Naples, all these authors have on their own way reinvented the city. It's between initial ostracism and almost superstitious idolization that the imaginary of Naples develops itself, fed by texts, History and cultural products. If a city can be rehabilitated by texts, we may claim that literature has still some value and legitimacy.
\end{abstract}

Mots-clés: Imaginaire de Naples, Regard interculturel, Licence artistique

Keywords: Naples imaginary, Cross-cultural gaze, Artistic licence 
ô voici l'animal qui n'existe pas.

[...] Certes il n'existe pas. Mais parce qu'ils l'aimaient un Animal pur naquit.

[...] Ils ne le nourrirent d'aucun grain,

mais uniquement de la possibilité d'être

Rainer Maria Rilke, Sonnets à Orphée, II, 4

Notre hypothèse est la suivante. La littérature, de par sa souveraineté, son droit inabrogeable à l'affabulation, sa faculté d'habiter les lieux, pourrait revaloriser, réhabiliter, resémantiser une ville en plein déclin, en l'occurrence Naples. Naples sera donc objet sémiotique, mieux notre templum. Dans l'antiquité les haruspices traçaient un carré imaginaire dans le ciel afin d'y étudier (con-templer) le passage des oiseaux, un cadre, une parcelle prégnante, de bon ou de mauvaise augure.

Pôle d'attraction à l'époque des Lumières, «le rendez-vous de l'Europe éclairée » (Fernandez, 1974 : 20), “point d'orgue du fameux 'Grand Tour' » (Schifano, 2007 : 117), " une grande capitale comme Paris", voire "la seule capitale d'Italie ", à en croire Stendhal, «toutes les autres grandes villes [étant] des Lyon renforcés » (Stendhal, 1826 : 317), la ville de Naples se voit désormais en plein déclin, " ostracisée » (Fernandez, 2008 : 52), dépréciée, stigmatisée, «spoliée » (Schifano, $2007: 336)$, «vouée, depuis la prétendue Unité avec Rome vaticane (100 000 habitants) comme capitale (1870), aux gémonies nationales puis internationales » (Schifano, 2007 : 50) par l'Italie du Nord, savoyarde, berlusconienne, charriant une réputation de rongée par la camorra, de regorgeant d'immondices, bref, incarnant ce qu'on appelle actuellement « la malanapoli » ${ }^{1}$. Car la littérature et la culture en général se taillent la part du lion dans l'exception napolitaine, toujours en antithèse avec d'autres villes, ce qui a suscité entre autre l'ouvrage intitulé Contre Venise de Regis Debray.

Celui-ci résume son coup de foudre pour Naples par la formule choc « [...] Je 'suis' Naples et non Venise. » (Debray, 1995 : 20) Contrairement au « style » (Debray, $1995: 31$ ) de Venise, «microcosme égocentrique » (Debray, $1995: 90)$ qui est totalement retranchée du monde par la « coupure sémiotique » (Debray, $1995: 24)$ dont fait office la lagune et qui accentue le sentiment d'ailleurs féerique, de «ville-jouet» (Debray, 1995: 29) ou « simulacre » (Debray, $1995:$ 30), le «tempérament (Debray, $1995: 31$ ) de Naples, en revanche, rappelle celui d'Anna Magnani dans Le Carrosse d'or. Et, comme si Debray

\footnotetext{
1 Jean Noël Schifano explique la vivacité de la camorra par le crime historique que la suprématie disproportionnée du Vatican a perpétré depuis deux siècles : "Naples dégradée de son rôle de capitale, l'une des plus importantes d'Europe, par un gouvernement romain qui n'arrivera jamais à ses fins - faire de Naples une ville de province soumise, une cité-bonzaï - et qui devrait, sans tarder, mettre en œuvre une véritable confédération italienne. Naples reprend illégalement, et aujourd'hui avec une puissance jamais atteinte, son rôle de capitale... » (Schifano, 2007 : 61-62.)
} 
illustrait déjà notre thèse, sa plume se met immédiatement à fabuler, à personnifier, dérogeant au style de l'essai :

La Parthénopéenne n'a pas besoin d'aller chez le coiffeur ni de vérifier son rimmel chaque quart d'heure dans une glace [...]. Putassière, charnue, généreuse, effrontée, avec ses klaxons, ses criailleries, ses obscénités, la truculente du Sud se jette à votre cou à vos risques et périls. La sensuelle tempête toutes tripes dehors - exhibant ses draps et ses petites culottes tendus au milieu de la rue (à Venise, le linge sèche dans la cour, convenances obligent). Elle beugle, sue, pue, piaille, provoque. (Debray, $1995: 31-32)$

Reprenant ensuite une métaphore éculée mais éminemment applicable à Naples, Debray parle de son « ventre » et de ses «entrailles» (Debray, 1995: 35), mais y ajoute une image plus personnelle, celle de «ville-gruyère » (Debray 1995: 36). Plus loin il se risque dans le champ de la faune marine, un «madrépore exsudant au fond de sa baie du pathos par tous ses pores. » (Debray $1995: 42$ )

S'il réfute radicalement le déclin de Naples, c'est encore par le biais d'une prosopopée : «Les vicissitudes ont raviné Venise, les ricorsi de Vico ont poncé le visage de Naples, comme si chaque spirale du temps remettait cette vitalité à neuf. 》 (Debray, 1995 : 36) Maints artistes ont exploité cette puissance revigorante de la ville. Ainsi le couple désœuvré du Voyage en Italie de Rossellini (1953), indifférents I'un à l'autre, voire aliénés par leur statut bourgeois, se ressaisit-il, se régénère-il dans la foule en liesse d'une procession napolitaine napolitaine.

II est vrai que la résilience ${ }^{2}$ de Naples, le fait que cette ville meurtrie, mise à sac, saignée à blanc, politiquement déchue se sauve toujours du déclin, tel un phénix qui renaît de ses cendres, se traduit depuis toujours dans toutes sortes d'emblèmes païens ou chrétiens, tels l'œuf de Virgile enfermé dans le Castel dell'Ovo censé assurer l'immortalité de la cité ou saint Janvier, patron de Naples, dont Alexandre Dumas invoquait déjà les intercessions multiples sous forme de litanie :

Les Normands ont régné sur Naples, mais saint Janvier les a chassés.

Les Souabes ont régné sur Naples, mais saint Janvier les a chassés.

Les Angevins ont régné sur Naples, mais saint Janvier les a chassés.

Les Aragonais ont usurpé le trône à leur tour, mais saint Janvier les a punis.

Les Espagnols ont tyrannisé Naples, mais saint Janvier les a battus.

Enfin, les Français ont occupé Naples, mais saint Janvier les a éconduits. (Dumas, 2006 : 194-195)

\footnotetext{
${ }^{2}$ Terme de psychanalyse qui signifie la capacité à surmonter un traumatisme (développé par Boris Cyrulnik).
} 
Mais c'est sans doute l'idolâtrie, «fusionnant culte des images et culte des morts », «Eros et Thanatos» (p.40) qui explique, selon Debray, cette vertu régénératrice, Venise s'avérant en revanche un « mirage d'où le sacré s'est envolé » (p.43) :

A Naples, où les anges de pierre sont souvent graves, comme ils doivent être, je redeviens idolâtre. Comme un enfant, un contemporain d'Homère, un pèlerin $d u$ Moyen Age. Tout prêt à accourir, à me pelotonner dans les bras des Vierges vermeil et des saints en argent mat, à me cacher sous leur chemise d'émeraudes et de diamants. J'y crois. [n.s.] A I'Image-Mère. Pourquoi ? Parce que les Napolitains y croient eux-mêmes. Ex-voto, talismans, amulettes. (Debray 1995 : 67-68)

C'est ce «j'y crois» que nous devons souligner, cette fiducie ${ }^{3}$ sur laquelle repose tout contrat littéraire, la willing suspension of disbeliefforgée par Coleridge en 1817. La superstition et l'affabulation sauvent du déclin car toutes deux reposent sur un croire et un faire croire.

Si Debray y croit, si Naples a pu le séduire, elle s'est en effet avérée terre d'asile par excellence pour de nombreux artistes: pensons au Caravage, fuyant à la fois la justice, après une rixe meurtrière à Rome, et la censure qui blâmait son excès de réalisme, notamment les pieds nus de saint Matthieu montrés « grossièrement » (Bellori 1991 : 21), ce qui ne l'empêcha pas de récidiver à Naples avec ses Sept CEuvres de Miséricorde où il représente "donner à manger aux affamés » par le biais d'« un vieillard qui passe sa tête entre les barreaux de sa prison et boit le lait d'une femme qui se penche vers lui, le sein nu » (Belori 1991 : 31) ; pensons au Divin Marquis qui, mêlé à plusieurs scandales dont celui « des petites filles » (Thomas, $2008:$ 11), vint lui aussi se réfugier à Naples; à Oscar Wilde, immigré dans une Naples à ses yeux moins hostile aux invertis ; à Gabriele D’Annunzio qui, couvert de dettes, y trouva un éditeur pour son roman L'Innocent ; à André Gide, qui rédigea L'Immoraliste (1902) à Ravello, sur la côte amalfitaine ; à Pasolini qui situa sa version filmique du Décaméron de Boccace en Campanie, parce que celle-ci lui offrait un patois plus authentique et un cadre plus rustique et populacier que l'aristocratique Toscane ; ou à JeanLuc Godard qui reprit le roman controversé Le Mépris de Moravia pour le situer dans l'inédite villa Malaparte à Capri, dont l'architecture («rationaliste », avec son toit solarium et ses escaliers trapézoïdaux quasi précolombiens) avait été conçue par l'auteur comme un autoportrait de pierre. Naples fut en outre lieu de révélation pour Wagner, qui trouva le jardin magique de Klingsor pour son Parsifal à Ravello, lieu de « renaissance » pour Montesquieu

\footnotetext{
${ }^{3}$ Jacques Fontanille et Claude Zilberberg déclinent la fiducie ou «fidélité sémiotique » en deux versions : «la confiance pour la relation intersubjective et la croyance pour la relation sujet/objet » (Fontanille \& Zilberberg, $1996: 198)$
} 
(Macchia, 1971: XIV), lieu d'élection car «métropole de l'opéra » (Fernandez, $1974: 21$ ) pour Stendhal, terre d'accueil pour un Dumas clandestin, s'étant vu refuser son visa à l'ambassade napolitaine à Rome par le régime bourbon et voyageant sous le nom de Guichard, peintre et pensionnaire de la Villa Médicis. Tous ces artistes et auteurs évoluent donc en sursis, en intrus, dans cette ville où la transgression est de rigueur, « où l'on ne peut vivre et jouir qu'en faisant de la transgression ludique la seule règle d'or du quotidien » (Schifano, 1984: 7). Naples, pépinière d'artistes, réceptacle d'écrivains en quête d'inspiration, de reconnaissance, voire de folie, s'avère à notre sens mériter pleinement et sans doute plus que toute autre le statut de «ville littéraire ».

II n'y a que la littérature qui puisse se faufiler dans tous les recoins de cette « villesfogliatelle » suivant l'audacieuse mise en abyme culinaire de Jean-Noël Schifano dans son savoureux Dictionnaire amoureux de Naples, sfogliatelle ou gâteau feuilleté digne de la madeleine proustienne :

La sfogliatelle a la forme de Naples, amphithéâtre où les maisons s'étagent elliptiquement les unes sur les autres, s'enchâssent les unes dans les autres, comme le fin ruban continu de pâte feuilletée. La sfogliatelle a la couleur blonde de la pierre dont est bâtie tout Naples, le tuf léger qui s'effrite et coule par vagues arrondies vers la mer, comme une demi-lune d'un rayon de miel au soleil d'avril. La sfogliatelle a la douceur de Naples, une douceur de dôme caressé d'azur, une douceur orientale, bombée, veloutée, enveloppante qui nous sollicite, nous séduit, nous absorbe et, jubilante métamorphose, nous fait oublier les minces rubans friables de notre moi occidental. (Schifano, $2007: 445$ )

Dans cette ville «feuilletée », «stratifiée », «étagée », «pliée », « striée » ou « rhizomatique » comme dirait Deleuze et dès lors «toujours démontable, connectable, renversable, modifiable, à entrées et sorties multiples, avec ses lignes de fuite » (Deleuze \& Guattari : 1980, 32), les trois artères centrales, le «Spaccanapoli » (le tranche-Naples) à l'époque gréco-romaine, la via Toledo, axe nord-sud datant de l'époque espagnole, rebaptisée via Roma par les fascistes, et récemment revenue à son vrai nom, et le corso Umberto I, éponyme d'un roi de la dynastie des Savoie, ne parviennent pas à asseoir leur pouvoir de centralisation, contrairement à d'autres axialités, de Vaux-le-Vicomte, illustre brouillon de Versailles pour Le Nôtre où la perspective fuit du château à l'horizon, jusqu'à Disneyland où, comme nous le rappelle Louis Marin, «Un chemin conduit directement au centre: 'la Grand'Rue Amérique'; Main Street USA. [...] l'axe le plus évident et le plus insistant de l' " utopie » [qui] dirige [...] le visiteur [...] de la réalité au fantasme ».(Marin, 2008 : 36) A Naples l'appareil d'Etat, est sans cesse déjoué, déconstruit, par le dédale des vicoli, ruelles transversales ou venelles ou par la fractalité de la baie elle-même. La topologie 
urbanistique est sans cesse perturbée par toute une série de facteurs dont maints écrivains se sont fait l'écho et qui traduisent la réalité d'un vécu napolitain : en profondeur, une espèce de bouillonnement magmatique, éruptions du Vésuve et tremblements de terre qui imposent une reconstruction perpétuelle de la ville et, en surface, le trafique-ballet des véhicules avec sa marée humaine («la végétation humaine » (Dupaty 1783)), la polysensorialité des bruits (les rires des enfants, les cris des vendeurs, les conversations sur les balcons et des odeurs de pierre lavique, senteurs, parfums divers ainsi que l'indifférenciation des classes qui superpose d'anciens palazzi à des "bassi », monolocaux « considérés comme insalubres » (Peyrefitte : 1952 : 65) habités par des familles entières, bref, ce que Schifano qualifie de « baroque existentiel » (Schifano, $2007: 33$ ).

Ville qui offre mille visages, ouvrant à des signifiés multiples comme le jardin de Kyoto décrit par Italo Calvino dans son récit intitulé «Les mille jardins » où « chaque pierre correspond à un pas, et à chaque pas correspond un paysage étudié dans tous ses détails, comme un tableau. » (Calvino, 1986 : 96) Or à Naples aucune intentionalité n'est à l'origine de ces innombrables points de vue, tout est aléatoire, s'engendre au gré des occasions, tout est vertigineux. Naples s'avère donc une ville-rhizome, un chaosmos plein de lignes de fuite à l'instar de l'Amsterdam de Deleuze, mais on pourrait avancer au même titre Lisbonne : «Amsterdam, ville pas du tout enracinée, ville-rhizome avec ses canaux-tiges, où l'utilité se connecte à la plus grande folie, dans son rapport avec une machine de guerre commerciale. » (Deleuze \& Guattari $1980:$ 24) La «sfogliatella » est un véritable défi à l'urbanisme comme le rhizome l'était à la botanique ou à la génétique.

II n'a que la littérature qui, par la « modélisation secondaire » qui la définit selon Jurj Lotman, soit à même de ressusciter une ville bien vivante mais jugée agonisante dans la perception de ses détracteurs. La modélisation secondaire que constitue le langage et ses virtualités semble inéluctable dans une ville où la " sémiosphère ", autre terme lotmanien, recèle un tel potentiel affabulatoire. (Lotman, 1999)

Même si la présence allemande fut essentielle pour la région (Goethe ${ }^{4}$, Gregorovius, Krupp, ce qui donna lieu à des formules telles que "Capri Sehnsucht» ou «Klein Deutschland»), nous avons voulu centrer nos propos sur la vision française de Naples. De Montesquieu (1729) à Sade (1775-1776), de Chateaubriand (1804) à Stendhal (1817), de Nerval (1835), Alexandre Dumas (1943), Lamartine (1852), Theophile Gautier (1852), Flaubert (1852) ou Maxime du Camp (1862), à Louis Barré (1877), c'est l'enthousiasme qui prime au contact de la ville mêlé de préjugés contre la barbarie du peuple enraciné dans un monde païen et attaché à des coutumes ancestrales. Au vingtième siècle c'est l'engouement

\footnotetext{
4 « A Naples chacun vit dans l'ivresse de l'oubli de soi. II en va de même pour moi. Je me reconnais à peine et il me semble être un tout autre homme. Hier, je pensais : 'Ou tu étais fou avant, ou tu l'es à présent' » (Goethe, in Schifano $2007: 119)$
} 
pur et simple qui prévaut : André Gide, Roger Peyrefitte, Dominique Fernandez, Jean-Noël Schifano.

Alberto Savinio, frère du peintre Giorgio de Chirico, développa une dualité intéressante : celle entre « autochtones » et “ullissides », c'est-à-dire entre indigènes d'une part et ceux qui « attirés par le chant jamais tari des Sirènes, affluent ici des points les plus éloignés du globe »(Savinio, 1988: 18, nous traduisons), de l'autre. Tous les chemins mènent à Naples, cet adage calqué sur Tous les chemins mènent à Rome est surtout valable à l'époque $d u$ "Grand Tour » au dix-huitième siècle, lorsqu'à la motivation «touristique » se mêle progressivement une motivation «artistique » ou «libertine $»^{5}$. Naples, ville baroque par excellence, attire les écrivains du Nord comme un îlot de liberté. Et la traversée des Alpes d'inaugurer cette levée des interdits. ${ }^{6}$

En général les regards d'écrivains sur l'hétérotopie sont soit target-oriented, comme on dit en théorie de la traduction, c'est-à-dire orientés vers une compréhension optimale du destinataire cible, soit source oriented, c'est-à-dire respectueux de la complexité ou de l'exotisme du pays visité, au risque d'intraductibilité. Ici, c'est la deuxième option qui l'emporte. Ainsi lors de son voyage en Italie entamé en 1728, après une soirée à la Scala de Milan, Montesquieu se dépouille-t-il aussitôt de sa condition de Français, voire se sentit-il Lombard (comme cela arrivera à Stendhal, dont on connaît l'épitaphe «Errico Beyle milanese, visse, scrisse, amò). Ce voyage donne en tous cas lieu à un dépaysement salutaire, entraînant une bonne leçon de relativisme : «Florence et Rome m'apprendront à voir Paris, car je ne l'ai point encore vu. » (Montesquieu, 1728, XIX). II faudrait relire L'Empire des signes de Roland Barthes (1970) qui au Japon se heurte à des signifiants vides, à l'exemption du sens, avec la possibilité de les remplir à sa guise ainsi que Amérique de Jean Baudrillard (Baudrillard, 1986) dont le regard achoppe, en revanche, à l'insignifiant radical, à la désignification, tant les stéréotypes anéantissent toute sémiose. A Naples on a, par contre, une hypertrophie du sens qui fascine, une polyphonie ou choralité qui invite à un dialogisme, qui fait parler de soi, pour ne pas dire, suscite des vocations d'ecrivains même jusqu'à l'agacement.

Parmi les « touristes » éclairés, Charles de Brosses est mis de mauvaise humeur par ce pays de canailles qui vous demandent sans cesse un pour boire et qui vous traitent de « poveri forestieri, c'est-à-dire, en langue vulgaire, les étrangers sont faits pour être volés. »

\footnotetext{
${ }^{5}$ On lira au sujet de la Méditerranée comme lieu de valorisation et d'investissement de sens, l'étude d'orientation sémiotique de Violi \&Tramonana, 2006.

6 Daniel Sangsue, dans un ouvrage intitulé Passages romantiques des Alpes, nous rappelle que «c'est aux Anglais que nous devons le mot et la notion de tourisme. "Avant que le goût ne se répande, aux dix-neuvième siècle chez tous les autres peuples d'Europe, les Anglais ont en effet été les précurseurs de cette manière de voyager à l'étranger motivée par le seul plaisir de découvrir du nouveau. Une de leurs traditions, très vivace aux dix-huitième siècle, voulait que les jeunes gens distingués effectuent à la fin de leurs études ce qu'on appelait le 'Grand Tour', c'est-à-dire une voyage sur le continent qui pouvait durer de vingt à trente mois et qui avait pour étapes les principales capitales européennes. » (Sangsue, $1990: 3$ )
} 
(de Brosses, 1739-1740: 211) Charles Mercier Dupaty, quant à lui, semble reconnaître aux Napolitains une espèce de seconde nature crapuleuse ou du moins insiste sur les glissements sémantiques de termes comme «misère », «filouterie », « débauche », voire « femme publique ", la débauche n'étant à ses yeux qu'une forme d'oisiveté. Et, quoique cette naturalisation des vices et des vertus puisse refléter une certaine condescendance de la part du civilisé qui exonère les barbares de leurs défauts, on peut également y lire une réelle empathie (Dupaty, $1785:$ 190)

De même, c'est la force rebelle des «lazzaroni » ou va-nu-pieds qui frappe Montesquieu, une plèbe «bien plus plèbe que les autres» (Montesquieu : 1728, 222). Cette ville tonitruante qui en soi ne l'intéresse pas avec ses ornements abondants, bizarres, « de mauvais goût » (Montesquieu, $1728: 211$ ), le fascine pourtant par son sol volcanique, ses eaux sulfureuses, « la limpidité aveuglante du ciel, le fond démoniaque, ésotérique, magique qui fermente dans le peuple, toujours en proie, jusqu'à la superstition et le fureur, à des forces aventureuses et mystérieuses. » (Montesquieu, 1728 : XXIV)

La magie du dépaysement qui suppose une resémantisation du connu, opère aussi sur l'Abbé de Saint-Non :

Quand on a tout peint et tout décrit (de Naples), il reste encore à rendre un effet magique qui existe dans l'air, qui colore tous les objets et qui fait que ceux mêmes que l'on connaît dans les autres climats, ne se ressemblent plus dans celui-ci et y deviennent nouveaux. (Peyrefitte, $1952: 86$ )

Le culte de la liquéfaction du sang de saint Janvier (début mai et le 19 septembre), évêque de Bénévent au $4^{\text {ième }}$ siècle, martyr décapité en 305 sous l'ordre du proconsul Timothée qui suivait le décret de son supérieur le consul Dioclétien, et dont les restes (sa tête et son petit doigt) sont enfermés au Duomo ainsi que son sang dans une ampoule, reflète bien la réalité napolitaine. Que les écrivains aient ressenti le besoin de relayer celle-ci est encore plus remarquable. Ils ont en quelque sorte entretenu la légende, préservé cette part de mystère qui constitue le charme de cette ville. D'une part Montesquieu, même s'il croit avoir assiste à la liquéfaction, en soupçonne bien vite l'imposture car son regard de naturaliste y décèle des causes objectives, physiques : le verre du reliquaire est embué par les baisers, il ne s'agit pas de sang coagulé mais d'un thermomètre dont le liquide, passant d'un lieu très frais à un lieu réchauffé par la multitude du peuple, par un grand nombre de chandelles et par les mains du prêtre, doit nécessairement se liquéfier. (Montesquieu, 1728 : 221) En outre il perçoit bien le but politique du miracle : un moyen de souder ces mendiants crédules, superstitieux, pour les soulager dans leur misère extrême. 
D'autre part, il est soudain pris par un moment d'hésitation où il est encore question de crédulité : « Je crois donc que les prêtres tombent eux-mêmes dans le piège ; ils ont vu la liquéfaction et ils ont cru qu'elle arrivait par miracle " (Montesquieu, $1728: 222$, n.s.). A force de baigner dans cette cérémonie, Montesquieu semble lui-même contaminé par sa fervente intensité religieuse. Se mettant à la place des fidèles, il sent la raison impuissante à expliquer le phénomène, comme si quelque chose d'occulte, de surnaturel lui échappait. D’où la conclusion : «Peut-être y a-t-il véritable miracle » (Montesquieu : 222).

Le marquis de Sade, dans son Voyage à Naples (1776), stigmatise toute l'entreprise comme une «simple expérience de physique », une "barbare ineptie », une "ridicule farce », « odieuse imbécillité » relevant des « ténèbres de l'ignorance » (Sade, $2008: 125)$ mais y voit à la fois, en tant que libertin éclairé, l'occasion de revaloriser le paganisme antique avec ses idoles par-delà les idolâtries chrétiennes. Autrement dit, Sade est à la fois choqué par le ridicule de ces cultes leur préférant ceux des païens mais en même temps profondément intrigué :

Après avoir passé le pont de la Madeleine, il ne faut pas manquer d'examiner à droite une statue de saint Janvier, une main levée sur le Vésuve et comme s'il lui commandait de s'arrêter. Ce monument fut élevé lorsque ce volcan cessa son éruption en 1767, mais on eut bien soin de ne poser la statue qu'après qu'elle eut cessé. II était, comme l'on croit, trop important de ne pas compromettre la réputation du saint, auquel le peuple eut une telle confiance [n.s.] que, dès qu'elle fut en place, il accourait par troupes, et montrant le derrière au Vésuve, il s'écriait: " Jette, jette, Vésuve, nous t'en défions! A présent, saint Janvier te le défend! » Je ne grossirais pas ma description de tels traits s'ils ne servaient à faire connaître l'esprit d'une nation dont l'esquisse des mœurs entre pour quelque chose dans mon entreprise. (Sade, $2006: 236)$

Alexandre Dumas, pour sa part, est exalté par le culte de saint Janvier au point d'y consacrer trois chapitres entiers dans son Corricolo, roman baroque s'il en est dont le titre renvoie à une espèce de tilbury où s'amassent toutes sortes de mendiants et de parasites.

Après avoir brodé autour des supplices infligés au martyr du $4^{\text {ième }}$ siècle, il décrit comment la chapelle du Trésor fut bâtie par de zélés notables napolitains en signe de reconnaissance publique à la suite de l'intercession du saint contre la peste qui sévit en 1527 et comment les peintres qui accoururent de partout pour l'orner de fresques (tels le Dominiquin) devinrent à leur tour des martyrs car furent évincés par les peintres locaux dont l'Espagnolet, à coup de tentative d'assassinat, d'empoisonnement ou de persécution : 
Alors les peintres napolitains se crurent délivrés de toute concurrence ; mais il n'en était point ainsi : un matin, ils virent arriver Gessi, qui venait avec deux de ses élèves pour remplacer le Guide son maître ; huit jours après, les deux élèves, attirés sur une galère, avaient disparu, sans que jamais plus depuis on entendit reparler d'eux ; alors Gessi abandonné perdit courage et se retira à son tour ; et l'Espagnolet, Corenzio, Lanfranco et Stanzione se trouvèrent maîtres à eux seuls de ce trésor de gloire et d'avenir, à la possession duquel ils étaient arrivés par des crimes. (Dumas, 2006 : 213)

Cet épisode des peintres étrangers, littéralement sacrifiés par les artistes locaux doit être lu en écho au propre vécu de Dumas, indésirable dans cette ville, incognito, clandestin, sauf à ruser, à négocier avec les règles et les lois. L'historique et le romanesque se mêlent encore indissociablement.

Le chapitre intitulé «Le miracle » corrobore le pouvoir de fascination de ce culte qui prime sur le vraisemblable scientifique. La fiducie, l'empathie, l'adhésion l'emportent même si Dumas n'a pas eu le temps d'assister à la cérémonie mais tient ses sources d'autres informateurs. D'où l'amplification, la magnification, l'hyperbole. La procession est décrite en termes de «torrent », de « flot », de « mer de têtes humaines », véritable meute chère à Deleuze, «pareille, enfin, à ces fleuves aux cours contraires, dont il est, grâce à leur double remous, presque impossible de distinguer la véritable direction. » (Dumas, 2006 : 219)

N'est-ce pas le propre du littéraire et de l'artistique que de s'en remettre au style, à la poétique et par là d'affabuler, de raconter des bobards, d'être mythomane ? Stendhal a pu nous faire croire qu'il était aux côtés de Napoléon dans sa Vie de Henry Brulard, tout comme Jacques-Louis David a pu dépeindre un Napoléon glorieux traversant le Grand SaintBernard dans la neige même si on sait que la réalité était bien plus prosaïque : le 14 mai 1800 il faisait doux, Napoléon franchit le col à dos de mulet et en simple redingote. La souveraineté de la littérature et de l'art en général réside dans cette mystification. La littérature et l'art doivent être considérées comme des «machines célibataires », selon l'expression de Deleuze et Guattari, c'est-à-dire privées de fertilité fonctionnelle, stériles, bref des dépenses inutiles, "machines " qui ont un droit inabrogeable à la «puissance du faux », à « l'autorité » de « l'imposture » selon la terminologie nietzschéennes cette fois.

Dumas insiste en tout cas sur la récidive du miracle au-delà de toute réfutation scientifique :

Maintenant, que le doute dresse sa tête pour nier, que la science élève sa voix pour contredire ; voilà ce qui est, voilà ce qui se fait, ce qui se fait sans mystère, sans supercherie, sans substitution, ce qui se fait à la vue de tous. La philosophie du dix 
huitième siècle et la chimie moderne y ont perdu leur latin : Voltaire et Lavoisier ont voulu mordre à cette fiole, et, comme le serpent de la fable, ils y ont usé leurs dents.

Maintenant, est-ce un secret gardé par les chanoines du Trésor et conservé de génération en génération depuis le quatrième siècle jusqu'à nous ?

Cela est possible ; mais alors cette fidélité [n.s.], on en conviendra, est plus miraculeuse encore que le miracle.

J'aime donc mieux croire [n.s] tout bonnement au miracle ; et, pour ma part, je déclare que j'y crois [n.s.]. (Dumas, $2006:$ 224)

J'aimerais paraphraser : c'est la longévité de la littérature qui est garante de son efficacité, de sa valeur. J'aime mieux y croire tout bonnement.

Roger Peyrefitte, un siècle plus tard, s'accommode d'un scepticisme de bon aloi :

Je songeai à cet art que l'Eglise témoigne, en Italie, de conserver ses vrais assises, c'est-à-dire la foi populaire. Elle a bien raison de ne pas faire analyser le contenu de l'ampoule. II faut en éloigner cette terrible science qui veut toujours tout savoir, autrement dit, tout détruire. (Peyrefitte, $1952: 24$ )

Or, ce que l'on veut montrer ici c'est qu'il faut un déclencheur : la fascination d'un mystère dans le cas de Saint Janvier ou d'un être mythique dans le cas de Napoléon pour que la fiducie s'installe. II n'empêche que le propre de la fiducie c'est d'inclure le croire et le non-croire, la conviction et le doute. Le faire croire, la suspension of disbelief de l'idolâtrie littéraire et superstitieuse accepte la marge de liberté de la méfiance (Sade), de la croyance aveugle (Dumas), de tout genre de scepticisme (Peyrefitte). C'est une des conditions pour que la liberté d'expression inconditionnelle soit garantie.

Revenons au Divin Marquis. Jugeant son propre guide touristique Voyage à Naples (1776) monotone - il s'agit en effet d'un carnet de voyage érudit où il consigne scrupuleusement ses impressions et il dénombre les églises jugées toutes médiocres -, Sade en recycla 20 ans plus tard des parties dans son Histoire de Juliette (1797). Comme si le dessein profond de son voyage était de relancer l'activité littéraire. Le passage du récit de voyage « ennuyeux », rébarbatif, répétitif, au récit de Juliette se fait dès lors moyennant des artifices romanesques. La séquence napolitaine référentielle se voit dotée d'une littérarité, d'une fonction esthétique, d'une fictionnalité. Or, à nouveau, cette émancipation de la valeur documentaire semble en l'occurrence possible grâce à ce terreau propice que constitue la Campanie. 
Dans la partie du Voyage consacrée à la nature environnante et aux ruines, le documentariste cède d'ailleurs déjà le pas au romancier malgré lui, s'abandonne à un relâchement voluptueux :

on y respire encore cet air mol et efféminé, qui dans ce climat délicieux, détruisait malgré soi les mœurs les plus pures et les principes les mieux établis. Virgile, Martial, Horace et Stace en ont assez vanté les douceurs pour qu'il ne soit pas possible de douter des délices qu'on y goûtait dans ses murs. [...] Vénus devait être la divinité d'une ville aussi corrompue. (Sade, $2008: 216-217$ )

Tout cela conforte notre thèse d'une ville et d'une région, propices aux épanchements littéraires et ici libidineux. Ainsi le déchaînement des éléments, la fougue de l'activité volcanique sera-t-elle relayée dans l'Histoire de Juliette par le débridement éruptif et enflammé de l'activité lubrique. Ainsi la consomption volcanique - «Les eaux [...] qui consument toutes promptement les matières qu'on y jette » (Sade, 2008 :192) - annonce-telle la consommation érotique sans reste, sans résultat, le flux stérile que d'aucuns ont comparé au flux du capital, et on pourrait lui ajouter la pure dépense de Bataille, les " débauches irréelles » (Barthes, $1971: 154)$ pour Roland Barthes. On le voit, le descriptif vire rapidement au narratif et l'épaisseur païenne de la région y est pour quelque chose. ${ }^{7}$

En revanche, la séquence de la cocagne toujours dans le Voyage à Naples, un échafaud où sont disposées des vivres, dont des oies encore vivantes, que le peuple peut se disputer, dès lors qu'elle est déjà tellement plastique et imagée en soi ${ }^{8}$, scène où la réalité dépasse la fiction tant la coutume semble invraisemblable, outrageusement théâtrale, est à première vue maintenue telle quelle dans la transposition romanesque. Or, tandis que le carnaval inaugurait dans les carnets un «tour » plutôt ennuyeux dans la ville, il sert en quelque sorte, dans I'Histoire de Juliette, de mise en appétit pour toutes les débauches, perversions et déviances qui suivront dans le périple de la jeune libertine.

Entre les carnets de voyage et le roman, il y a une nette évolution du dyphorique à l'euphorique, de l'évaluatif au participatif. Dans le roman la séquence est en outre précédée

\footnotetext{
7 A l'inverse, le descriptif peut réémerger ironiquement pour atténuer le narratif dans Histoire de Juliette: «Reposées de cette fatigue, nous songeâmes à continuer notre tournée des environs de Naples, du côté du levant : si ces descriptions ne vous déplaisent pas, j'en entremêlerai celles de mes luxures. Cette variété amuse ; elle est piquante : si jamais ces récits s'imprimaient, le lecteur, dont l'imagination [n.s.] est échauffée par les détails lubriques qui parsèment cette narration, ne serait-il pas enchanté d'avoir à se reposer quelquefois sur des descriptions plus douces, et toujours marquées du sceau de la plus exacte vérité. L'œil du voyageur, fatigué des points de vue pittoresques qui l'occupent en traversant les Alpes, aime à s'arrêter sur les plaines fertiles qu'il trouve au bas des monts, où la vigne, agréablement enlacée à l'ormeau, semble toujours, dans ces belles contrées, indiquer la nature en fête. » (Sade, $1797: 1067)$ Sade prend «imagination » au sens de " sensualité » des anciens comme alibi de son inéluctable virement du descriptif au narratif, de sa fougue narrative impossible à contenir plus longtemps.

${ }^{8}$ Sans aller jusqu'à dire comme Dominique Fernandez, «La rue, à Naples, fait une concurrence déloyale à la littérature. Rien à faire : l'écrit y sera toujours le parent pauvre du vécu. » (Fernandez, 2008 : 54)
} 
de préambules qui mettent en place la focalisation: le balcon du palais royal, la petit compagnie buvant du chocolat où des figures historiques (le roi Ferdinand en maître de cérémonie et la reine Charlotte) côtoient des êtres de papier qui décident « sadiquement » du sort des victimes.

Allons, belles dames, nous dit Ferdinand, donnez vos ordres : en raison du plus ou moins de rigueur, du plus ou moins de police que je mets à la célébration de ces orgies, je puis faire tuer six cents hommes de plus ou de moins : prescrivez-moi donc ce que vous désirez à cet égard... - Le pis, le pis ! répondit Clairwil ; plus vous ferez égorger de ces coquins, et plus vous nous amuserez. - Allons", dit le roi, en donnant bas un ordre à l'un de ses officiers ; puis, un coup de canon s'étant aussitôt fait entendre, nous nous avançâmes sur le balcon. II y avait un peuple excessivement nombreux sur la place; alors nous découvrîmes toute la perspective. (Sade, 1797 : 1085-1086)

Les deux passages mériteraient une étude comparative. En voici quelques bribes. L' « amour pour le vol » du «peuple sauvage » (Sade, $2008: 30$ ) des carnets de voyage devient «son excessif amour pour le vol » (Sade, $1797: 1086)$. Cette « effrayante scène » qui « finit quelquefois tragiquement » (Sade, 2008, 30) dans la première rédaction, «finit toujours plus moins tragiquement »(Sade, $1797: 1087)$ dans la seconde.

La fougue narrative de Sade dans la description de la mêlée impitoyable entre concurrents et quartiers de bœuf lui fait en outre exagérer le nombre de vies qu'exige l'écroulement de la décoration sur les assaillants

\section{Voyage en Italie}

Huit minutes suffisent à la destruction totale de l'édifice ; et sept ou huit morts et une vingtaine de blessés qui souvent meurent après, est ordinairement le nombre des héros que la victoire laisse sur le champ de bataille. (Sade, $2008: 30$ )

Histoire de Juliette

Mais cette fois, d'après nos désirs, par les soins cruels de Ferdinand, quand le théâtre fut chargé, quand on crut qu'il pouvait bien y avoir sept ou huit cents hommes dessus, tout à coup il s'enfonce, et plus de quatre cents personnes sont écrasées (Sade, $1797: 1086-1087)$

C'est le paganisme profond avec sa liberté de mœurs que Sade veut mettre au jour chemin faisant dans l'Histoire de Juliette, pour laisser libre cours à sa licence littéraire et libidinale, bref au scénario sadique même. Car non seulement la vue des animaux maltraités 
et agonisants offerte à la joie des foules constitue une mise en abyme de la vue des gueux égorgés offerte à l'amusement de la cour mais cette dernière sert d'amorce, de mise en appétit, presque de légitimation à l'orgie qui suivra et qui, comme pour prolonger l'ignominie en pratique purement sadique, organisera le supplice des épouses des infortunés qui viennent de périr sous leur yeux :

allons, foutons, faisons-nous foutre, vexons, tourmentons, supplicions, et que nos têtes, embrasées par le spectacle qui vient de nous être présenté, raffinent à la fois les cruautés et les luxures... (Sade, $1797:$ 1088)

D'autre part, la reprise littéraire de la description de la statue du satyre accouplé à une chèvre au musée de Portici, ne subit que peu d'ajouts, puisqu'elle témoigne déjà d'une extrême liberté païenne. On sait depuis que tous ces Priape, satyres et silènes n'étaient pas l'encensement de la débauche perpétuelle chez les Romains mais plutôt des symboles de fertilité nullement obscène. C'est l'époque moderne, en les arrachant aux murs de Pompéi, en les cachant ou les atténuant dans les copies, bref en les censurant qui les ont rendus lascifs, obscènes.

\section{Voyage en Italie}

Mais le morceau le plus secret et le plus singulier de toute cette collection nombreuse se conserve chez le sieur Canart, sculpteur du roi. C'est un groupe de marbre d'environ un pied et demi de hauteur dont le sujet est un satyre jouissant d'une chèvre. Il est difficile de mettre plus d'âme et d'expression que l'artiste n'en a mis tant dans tous les mouvements et les muscles du satyre, que dans ceux de la chèvre. Sa langue sur le bout de ses lèvres exprime tout le plaisir qu'elle sent, et la manière vive dont le satyre la tient par sa petite barbe ne sert pas peu à lui donner de la chaleur. Tout est en action dans ce beau morceau, tout est en feu; la plus exacte pureté de style le caractérise. Mais on ne permet pas à tout le monde d'en juger, et la sévérité de mœurs du marquis Tanucci a obtenu du roi de n'en accorder que très difficilement la permission. (Sade, $2008: 264-265)$

\section{Histoire de Juliette}

Je distinguai parfaitement, entre autres, un morceau superbe représentant un satyre jouissant d'une chèvre : il est impossible de rien voir de plus beau... de mieux fini. "Cette fantaisie est aussi agréable qu'on la trouve extraordinaire, nous dit Ferdinand. Elle est, nous dit-il, encore fort en usage dans ce pays-ci ; en qualité de Napolitain, j'ai voulu la connaître, et je ne vous cache pas qu'elle m'a donné le plus grand plaisir. - Je le crois, dit Clairwil ; cette idée m'est venue mille fois dans la vie, et je n'ai jamais désiré d'être homme que pour l'éprouver. - Mais une femme peut très bien se livrer à 
un gros chien, dit le roi. - Assurément », répondis-je, de manière à fairecroire [n.s.] que je connaissais cette fantaisie (Sade, $1797: 1068$ )

Or, à nouveau, le roman relaye le plaisir scopique par une promesse de plaisir vécu, aussi perverse soit-il.

L'absence d'inhibition aucune propre à la fiction se mesure au fait que cette même statue soit passée sous silence, frappée d'omertà dans le traité historico-archéologique intitulé Musée secret de Louis Barré (1877) et certains détails prégnants, saillants, sensiblement atténués dans les gravures de Henri Roux qui l'accompagnent: " nous ne dirons point un mot de la seconde figure, qui représente un groupe de marbre de Paros. [...] Dépêchons-nous de tourner la page. »(Barré, 1877 : 221)

Sade nous montre que la littérature a encore une fonction, ce pouvoir de dépasser les limites que Platon situait déjà dans le langage (Deleuze, 1969 : 10), de parler sans être muselé, bâillonné. Sans doute avait-il déjà pressenti les esthétiques qui prônent la souveraineté de la littérature, son autonomie, qu'il s'agisse de « donner un sens plus pur aux mots de la tribu », de dire «l'absente de tous bouquets » ou d'écrire un livre «sur rien ». Barthes a insisté sur cet irréalisme sadien : "Ce qui se passe dans un roman de Sade est proprement fabuleux [n.s], c'est-à-dire impossible ; ou plus exactement, les impossibilités du référent sont tournées en possibilités du discours [...] 》 (Barthes, $1971: 131$ )

Naples étant déjà un roman, elle s'avère une ville propice au fictionnel ${ }^{9}$, à l'affabulation, à la superstition. Si la littérature a encore une valeur cela pourrait être de redresser la vision actuelle tronquée d'une ville par des regards multiples, de lui redonner une épaisseur, de la revivifier. D'ailleurs, Naples, par un effet de résilience, a toujours pu atténuer ses plaies en les mettant en scène, en faisant foi à la littérature, à la fiction. La superstition et la littérature ont ceci en commun qu'elles nous font croire à l'impossible.

On pourrait poursuivre notre diachronie en montrant que le Voyage en Italie de Chateaubriand (1803-1804) relève d'emblée d'une mise en discours romancée, romantique, enflée de métaphores et d'hyperboles et que Théophile Gautier, dans son récit fantastique Arria Marcella (1852) se livre à l'affabulation pure, en fantasmant à partir d'un corps en creux dans la lave. Mais l'espace qui nous est imparti ne nous le permet pas. Concluons par un autre topos éminemment napolitain, celui de la statue du Christ voilé (1753) de Giuseppe Sanmartino, sculpteur baroque par excellence. Le prodige de cette sculpture en marbre consiste dans le suaire drapé d'une telle transparence qu'il fait ressortir, au lieu de cacher, les chairs et les traces du supplice.

\footnotetext{
9 "Ce peuple enthousiaste, très sceptique au fond. Emploi abusif du conditionnel. On ne dit pas 'Quelle est cette rue ?... » «Quelle heure est-il ?... »mais serait. Je demande à une brave femme si une bambina qui se trouvait là, était sa fille. «Ce serait ma fille », répondit-elle. » (Roger Peyrefitte, o.c., p. 77)
} 
La littérature doit à la fois voiler le réel et le dévoiler, s'émanciper du référentiel et le réinventer, le resémantiser au risque de ne pas être rentable, d'encourir l'opprobre, la censure, la stigmatisation. Le littéraire est un droit que certains régimes totalitaires, frileux, privés de sens de l'humour oppriment sous prétexte d'offense à la religion ou à la morale, ou d'autres régimes utilitaristes, vouent aux gémonies à cause de sa gratuité, de sa frivolité. II faut donc revendiquer ce droit à exercer l'écriture au figuré, le mythe, la dépense inutile. Si la littérature peut sauver une ville du déclin, nous, en tant que les citoyens du monde, devons sauver la littérature, lui faire confiance, croire en elle-même quand elle ment, faire foi en son avenir :

II faisait une de ces heureuses journées si communes à Naples, où par l'éclat du soleil et la transparence de l'air les objets prennent des couleurs qui semblent fabuleuses [n.s.] dans le Nord, et paraissent appartenir plutôt au monde du rêve qu'à celui de la réalité. (Gautier, $1981: 239)$ 


\section{Bibliographie}

ABBE DE SAINT-Non, Le voyage pittoresque ou Description du royaume de Naples et de Sicile, 17811786.

BARTHES, Roland, L'Empire des signes. Paris : Seuil, 1970.

BARTHES, Roland, Sade, Fourier, Loyola. Paris : Seuil, 1971.

BARRÉ, Louis, Musée secret. Paris, 1877 (Herculanum et Pompéi. Tome VIII, Recueil général des peintures, bronzes, mosaïques, etc. découvertes jusqu'à ce jour et reproduites d'après Le antichità di Ercolano, il museo Borbonico et tous les ouvrages analogues, augmenté de sujets inédits gravés au trait sur cuivre par H.Roux Ainé et accompagné d'un texte explicatif par M.L.Barré).

BAUDRILlARD, Jean, Amérique. Paris : Grasset \& Fasquelle, 1986 "poche »

BellorI, Giovan Pietro, Vie du Caravage. (trad. de l'italien par Brigitte Pérol) (Vite di pittori, scultori e architetti moderni di Bellori, ed. Evelina Borea e Giovanni Previtali, Torino : Einaudi, 1976), Paris : Gallimard, 1991.

BROSSES Charles de, Lettres familières écrites en Italie, 1739-1740. In : VAUTIER, 2007.

CALVINO, Italo, «Les mille jardins », in Collection de sable. Paris : Seuil, 1986 (trad. Jean-Paul Manganaro) ( «I mille giardini », in Collezione di sabbia. Milano : Garzanti, 1984).

Deleuze, Gilles, Logique du sens. Paris : Minuit, 1969.

Deleuze, Gilles \& GuatTARI, Félix, Mille Plateaux. Paris : Minuit, 1980.

DumAs, Alexandre, Le Corricolo. Paris : Desjonquères, 2006.

DUPATY, Charles Mercier, Lettres sur l'Italie, à Rome. 1785. In : VAUTIER, 2007

FERNANDEZ, Dominique, «Tronches napolitaines ». In : Le Nouvel Observateur, 17-23 juillet 2008

Fernandez, Dominique, Porporino ou les mystères de Naples. Paris : Grasset, 1974.

FonTANILle, Jacques \& ZILberberg, Claude, Tension et Signification. Liège : Mardaga, 1996.

GaUtier, THEOPHILE, Arria Marcella, Souvenirs de Pompéi (1852). In : Récits fantastiques. Paris : Garnier-Flammarion, 1981.

LOTMAN, Juri, Le sémiosphère (1984). Limoges : PULIM, 1999.

MACCHIA Giovanni, prefazione a Montesquieu, Viaggio in Italia. Bari : Laterza, 1971 (CEuvres complètes, «Pléiade » vol.1 (Roger Caillois), vol II (André Masson), 1949)

MARIN, Louis, «Dégénérescence utopique : Disneyland». In : Le pouvoir dans ses représentations, Catalogue publié à l'occasion de l'exposition. Galerie Colbert, 29 mai-26 juillet. Paris : INHA, 2008.

MONTESQUIEU, Lettre à Madame Lambert, 26 déc.1728 In : MACCHIA, 1971

Peyrefitte, Roger, Du Vésuve à l'Etna. Paris : Flammarion, 1952.

SADE, Le Marquis de, Voyage à Naples, Paris, Payot \& Rivages, 2008.

SADE, Le Marquis de, Histoire de Juliette, 1797. In : CEuvres complètes, Paris : Gallimard, «Pléiade ", tome 3 (éd. Michel Delon).

SANGSUE, Daniel (textes réunis et présentés par), "Le Grand-Saint-Bernard, haut lieu romantique ». In : Passages romantiques des Alpes. Lausanne : Favre, 1990.

SaVINIO, Alberto, Capri (1926). Bari : Adelphi, 1988.

SCHIFANO, Jean-Noël, «Alessandro Dumas, Napoletano » 1984, Préface à DuMAS, 2006.

SCHIFANO, Jean-Noël, Dictionnaire amoureux de Naples. Paris : Plon, 2007.

StENDHAL, Rome, Naples, Florence (1826). Paris : Gallimard (éd. Pierre Brunel), 1987, « folio classique », 20 février 1817.

THOMAS, Chantal, Préface à Sade, 2008. 
VAUTIER, Dominique, Tous les chemins mènent à Rome. Voyages d'artistes du $16^{\text {ième }}$ au $19^{\text {ième }}$ siècle, catalogue de l'exposition au Musée Communal d'Ixelles, 11 octobre 2007 - 27 janvier 2008, dans le cadre du festival Europalia Europe 2007, Fonds Mercator, Europalia.Europe : Musée communal d'Ixelles, 2007.

VIOLI, Patrizia \& TRAMONTANA, Andrea, « Méditerraneo : idendità e border crossing fra terra e mare. In : Senso e metropoli. Per una semiotica posturbana (a cura di Gianfranco Marrone e Isabella Pezzini). Roma : Meltemi, 2006, pp. 109-130. 